\title{
sourmash: a library for MinHash sketching of DNA
}

\section{Titus Brown ${ }^{1}$ and Luiz Irber ${ }^{1}$}

DOI: $10.21105 /$ joss. 00027

\section{Software}

- Review ¿

- Repository ca

- Archive ${ }^{\top}$

\section{Licence}

Authors of JOSS papers retain copyright and release the work under a Creative Commons Attribution 4.0 International License (CC-BY).
1 University of California, Davis

\section{Summary}

sourmash is a toolbox for creating, comparing, and manipulating MinHash sketches of genomic data.

MinHash sketches provide a lightweight way to store "signatures" of large DNA or RNA sequence collections, and then compare or search them using a Jaccard index. MinHash sketches can be used to identify samples, find similar samples, identify data sets with shared sequences, and build phylogenetic trees (Ondov et al. 2015).

sourmash provides a command line script, a Python library, and a CPython module for MinHash sketches.

\section{References}

Ondov, Brian D, Todd J Treangen, Adam B Mallonee, Nicholas H Bergman, Sergey Koren, and Adam M Phillippy. 2015. "Fast Genome and Metagenome Distance Estimation Using Minhash." bioRxiv. Cold Spring Harbor Labs Journals, 029827. doi:10.1101/029827. 